\title{
XI.
}

Aus der II. medizinischen Klinik der königl. Universität Budapest (Direktor: Prof. E. Jendrássik).

\section{Über einen Fall von Myasthenia gravis pseudoparalytica mit positivem Muskelbefund.}

Von

\section{Dr. Josef Csíky.}

(Mit 5 Abbildungen.)

Obwohl S. Wilks ${ }^{1}$ ) im Jahre 1877 der erste war, der symptomatologisch einen zur Myasthenie gehörigen, tödlich verlaufenden Fall mit negativem Sektionsbefund veröffentlichte, so knüpft sich die Erkennung dieser Krankheit als eines selbständigen Krankheitsbildes an die im Jahre 1878 erfolgte Veröffentlichung von 3 Fällen durch W. Erb. ${ }^{2}$ ) Auf rein klinischer Grundlage wies er auf den "neuen, wahrscheinlich bulbären Symptomenkomplex" hin, der sich besonders durch seinen eigenartigen Verlauf und seine Tendenz zur Heilung von der gewöhnlichen progressiven Bulbärparalyse unterschied.

Erst im Jahre 1887 erfolgte die nächste Veröffentlichung eines Falles mit Autopsie durch Oppenheim ${ }^{3}$ ) mit gänzlich negativem Sektionsbefund, so dass er das Krankheitsbild mit dem Namen „Bulbärparalyse ohne anatomischen Befund" bezeichnete. Unter den in den nächsten Jahren in grösserer Zahl veröffentlichten Arbeiten verdient die vou Goldflam ${ }^{4}$ ) besonderer Erwähnung, der zuerst auf die eigenartige Ermüdbarkeit der Muskeln als etwas von der Lähmung Verschiedenes hinwies und zeigte, dass in manchen Fällen sogar Heilung eintreten kann; auch wies er als erster darauf hin, dass ausser den bulbären Symptomen dieselben Erscheinungen der Ermüdbarkeit auch an den Extremitäten ohne jede Muskelatrophie nnd Degeneration auftreten können. Eine andere wichtige Veröffentlichung ist die von Jolly ${ }^{5}$ ), in der er das unter dem Namen "myasthenische Reaktion" bekannte Verhalten der Muskulatur gegenüber dem elektrischen Strom beschreibt.

Die in den Arbeiten von Strümpell ${ }^{6}$, Hoppe ${ }^{7}$, Eisenlohr ${ }^{8}$ ), Senator ${ }^{9}$, , Shaw ${ }^{10}$ ), Dreschfeld ${ }^{11}$ ), Somdorfi2), Schüle ${ }^{13}$ ), Ray-

Deutsche Zeitschrift f. Nervenheilkunde. 37. Bd. 
mond $\left.{ }^{14}\right)$ u. a. veröffentlichten Fälle ergaben alle bei der Sektion einen negativen Befund. Diese und viele ohne Sektion beschriebenen Fälle trugen aber dazu bei, dass sich ein streng umgrenztes und charakteristisches Krankheitsbild aufstellen liess, das klinisch leicht zu erkennen war, in ätiologischer und pathologisch-anatomischer Hinsicht aber ganz im Dunklen blieb. So konnte dann im Jahre 1901 Oppenheim ${ }^{15}$ ) in seiner ausgezeichneten Monographie ein klares Krankheitsbild entwerfen, ohne aber die der Krankheit zugrunde liegende pathologische Veränderung angeben zu können.

Die veröffentlichten Fälle erwiesen es klar, dass das Leiden kein speziell bulbäres ist, sondern dass es auch die übrigen Körperteile, resp. Muskeln ergreifen kann, wobei bei den einzelnen Fällen an verschiedenen Muskeln die ersten Symptome der Erkrankung auftreten können, aber wohl am häufigsten in den bulbären Gebieten. Schon minimale Schwäche einzelner Augenmuskeln, des M. Ievator palpebrae sup., der Gaumenmuskulatur verursachen Symptome wie Doppelsehen, Senkung der Augenlider, Schluckbeschwerden, die dem Kranken natürlich auffallen und ihn zunächst ohne andere Erscheinungen und Beschwerden zum Arzte führen. Die in den Extremitäten eventuell ebenfalls schon vorhandene Schwäche ist um diese Zeit noch so gering, dass sie vom Kranken gar nicht beachtet wird oder höchstens in einer allgemeinen Mattigkeit ihren Ausdruck findet.

Es ist daher nichts Auffallendes, dass die meisten Autoren zuerst in einer Erkrankung des zentralen und auch des peripheren Nervensystems die anatomische Grundlage dieser Erkrankung suchten. Besonders das verlängerte Mark wurde immer und immer wieder untersucht. Die dabei gewonnenen Resultate waren entweder absolut negativ, oder es fanden sich im Zentralnervensystem an manchen Stellen frische, mikroskopische Blutungen, Chromatolyse in den Ganglienzellen der Kerne der Kopfnerven, minimale Degenerationen in verschiedenen Wurzelgebieten nach Marchi, auffallende Dünnheit einzelner Wurzeln und Wurzelfasern, mit einem Wort lauter Veränderungen, die wegen ihrer Geringfügigkeit nicht als Krankheitsursache angenommen werden konnten.

Bei diesen negativen Befunden gewann langsam jene Anschauung an Bedeutung, die das ganze Krankheitsbild als eine Autointoxikation auffassen wollte, welche durch pathologisehe Stoffwechselprodukte, sog. Ermüdungsstoffe, oder durch die Wirkung anderer Toxine, besonders aber durch die Produkte der in vielen Fällen persistierenden Thymus und verschiedener Tumoren verursacht wird. Auffallend häufig war auch eine kongenitale Missbildung an verschiedenen Teilen des Körpers nachweisbar, die auf eine angeborene Minderwertigkeit des 
Organismus hinzuweisen schien und die geeignet war, die letztere Auffassung von der Toxinwirkung dadurch zu nnterstützen, dass ein von Geburt ans minderwertiger Organismus viel leichter durch toxische Stoffe geschädigt wird, ohne Unterschied in Bezug darauf, ob diese Stoffe im Körper selbst erzeugt werden (Autointoxikation), oder ob durch auswärtige Gifte die Intoxikation verursacht wird.

Im Jahre 1901 publizierte Weigert ${ }^{16}$ ) als erster einen von Laqueur ${ }^{16}$ ) beobachteten typischen Fall von Myastheniè, bei dem die Sektion ein positives Resultat ergab, indem neben einer Thymusgeschwulst mikroskopische Veränderungen in den Muskeln nachweisbar waren. Bei normalem Nervensystem fand er eine stark vergrösserte und geschwulstartig veränderte Thymus, bestehend aus lymphoiden und wenig epitheloiden Zellen mit typischen Hassalschen Körperchen. Dieselben Zellen fand er auch mikroskopisch in den makroskopisch ganz normal aussehenden Skelettmuskeln sowie anch im Herzmuskel. Es waren das zerstreut und gruppenweise angeordnete lymphoide Zellen zwischen den einzelnen Muskelfasern im Perimysium int., ohne jegliche Entzündungserscheinungen, ohne polynukleäre Leukocyten und ohne Fibroblasten mit neugebildetem Bindegewebe. Da die Zellen in jeder Beziehung den in der Thymus gefundenen Tumorzellen glichen, erklärte er sie als mikroskopisch kleine Metastasen der Thymusgeschwulst. Trotzdem brachte er das ganze Bild in keinen ursächlichen Zusammenhang mit der Krankheit, da er es nicht für wahrscheinlich hielt, dass so geringe Muskelveränderungen ein so schweres Krankheitsbild verursachen könnten. Darum glaubt er eher als Ursache intermediäre Stoffwechselprodukte annehmen zu können, die von der persistierenden und krankhaft veränderten Thymus secerniert würden. Seit dieser Veröffentlichung von Weigert erschienen weitere Befunde, gestützt teils auf Sektionen, teils auf Untersuchungen von den Lebenden exzidierten Muskelstückchen.

Im allgemeinen lassen sich diese Befunde in 2 Gruppen einteilen: 1. in solche, bei denen eine primäre Geschwulst irgendwo im Körper vorhanden war und wo der im Muskel erhobene Befund als Metastase der Geschwulst gedeutet wird; 2. in Fälle, bei denen ausser dem charakteristischen Muskelbefund nichts anderes nachweisbar war. In den Fällen beider Gruppen aber war ein auffallend häufiger Befund eine Persistenz der Thymusdrüse. Zu der ersten Gruppe gehören ausser dem oben erwähnten Fall von Weigert noch die Fälle von Goldflam ${ }^{17}$ ) und $\operatorname{Hun}^{18}$, während zu der zweiten Gruppe die Fälle von Steinert19), Link $\left.{ }^{20}\right)$, Boldt21), Burr $\left.{ }^{22}\right)$, Buzzard ${ }^{23}$ ) (5 Fälle), Knoblauch ${ }^{24}$ ) Frugoni ${ }^{25}$ ), Marburg ${ }^{26}$ ), Osann ${ }^{27}$ ) und Mandelbaum u. Celler ${ }^{28}$ ) zu rechnen sind. 
Alle durch diese Autoren erhobenen Befunde stimmen im. allgemeinen miteinander überein, so dass ich auf sie im einzelnen nicht näher eingehen will. Es fanden sich immer im Perimysium int. und zwischen den einzelnen Muskelfasern Anhäufungen von "lymphoiden" Zellen, die man auch wegen ihrer regelmässig um kleine Gefässe sich gruppierende Anordnung perivaskuläre kleinzellige Infiltration nannte.

Da jeder neve mit Muskelbefund einhergehende Fall einen positiven Beweis bildet für eine Mitbeteiligung der Muskeln an der Krankheit, so möchte ich hier einen typisch verlaufenen Fall mitteilen, bei dem die mikroskopische Untersuchung einzelner Muskeln einen ähnlichen positiven Befund ergab.

E. Sziderits, 21jähr. Feldarbeiterin, aufgenommen in die Nervenklinik am 26. Juli 1904, gestorben am 25. Juli 1907.

Familienanamnese bietet nichts Bemerkenswertes. Eltern waren nicht verwandt. Von 11 Geschwistern leben drei, eine Schwester starb an Lungentuberkulose, die übrigen Geschwister sind im Kindesalter gestorben.

Nach Angabe der Patientin war sie immer gesund and kräftig, hatte keine Kinderkrankheiten, keine Diphtherie oder andere Infektionskrankheiten durchgemacht. Menstruation mit 12 Jahren, bis zur jetzigen Krankheit immer regelmässig. Nur seit Beginn der Krankheit blieb sie ôfters 2-3 Monate aus, dann stellte sie sich mit starken Schmerzen ein.

Ihrem jetzigen Leiden ging weder eine akute Erkrankung noch Erlrältung voraus. $\mathrm{Za}$ Beginn des Jahres 1902 bemerkte sie, dass ihre Arme bei der Arbeit auffallend schnell müde wurden; nach einem halben Jahr fühlte sie ein ähnliches Schwächegefühl bei längerem Gehen in den Beinen, wozu sich allmählich eine Ermüdung bei längerem Sprechen, zeitweises Herabsinken beider Augenlider und Doppelsehen gesellten. Auch das Schlucken wurde ihr manchmal beschwerlich und es geschah ofters, dass ihr Flüssigkeit beim Schluckversuch durch die Nase regurgitierte. Besonders auffallende Schmerzen soll sie niemals gehabt haben. Schon bei leichter Arbeit fühlte sie sich matt; diese schnelle Ermüdbarkeit nahm mit der Zeit immer mehr zu, so dass Patientin zuletzt gar nicht mehr arbeiten konnte; sie wurde bettlägerig und konnte sich anch im Bette kaum bewegen. Dieser Zustand dauerte 6-8 Wochen, wonach es ihr wieder besser ging; sie begann von nenem zu gehen, die Ermüdbarkeit stellte sich aber nach der kleinsten Anstrengung immer wieder ein, so dass sie arbeitsunfähig war. In diesem Zustand suchte sie unsere Klinik auf.

Von anderen Beschwerden muss noch besonders hervorgehoben werden die hartnäckige Schlaflosigkeit, gedrückte Stimmung, die schnelle Erregbarkeit des Gemüts und manchmal Herzklopfen. Appetit, Stuhl normal, keine Urinbeschwerden. Angeblich Amor lesbicus gegenüber einer Gefährtin, später auch gegen andere.

Status praesens. Mittelgrosse, kräftig gebaute Patientin in gutem Ernährungszustand. Knochensystem, Gelenke normal. Haut blass, so auch die sichtbaren Schleimhäute. Keine Drüsenschwellung. Lunge normal. Herz innerhalb normaler Grenzen, Herztöne rein. Puls ebenfalls normal, in der Ruhe 82, stieg aber nach psychischen Reizen and körperlichen An- 
strengungen sehr rasch bis auf 120 in der Minute. Bauchorgane ohne Befund. Urin enthält kein Eiweiss oder andere pathologische Bestandteile.

Das Gesicht der Kranken macht den Eindruck starker Müdigkeit; die Falten des Gesichts, besonders die Nasolabialfalten, sind beiderseits fast ganz verstrichen. Die Augenspalte ist verengt, indem das obere Augenlid tiefer steht als normal. Nach mehrmaligem. Öfnen und Schliessen der Augen, so auch nach 10-15 Seitwärtsblicken schliessen sie sich fast vollständig, trotzdem Patientin sich die grösste. Mühe gibt, sie offen zu halten. Nach einer kurzen Pause von 1-2 Minuten kann Patientin die Augen wieder öffnen. Fast immer aber bleibt beim Schliessen der Augen zwischen den Lidern ein kleiner offener Spalt. Pupillen sind gleichweit, rund, reagieren auf Licht und Konvergenz gut und zeigen gar keine Ermüdungserscheinungen. Augenbewegungen sind nach allen Richtungen frei. Konvergenz normal. Nach längerem Sehen nach rechts gibt sie an, manchmal doppelt zu sehen. Geringer Exophthalmus. Kein Nystagmus. Cornealreflex vorhanden. Die Beweglichkeit der Stirnmuskulatur ist sebr beschränkt; Patientin kann ihre Stirn nor in sehr geringem Maße runzeln. Sie hält den Mund fast immer etwas offen, trotzdem die Nasenatmung ganz frei ist. Der rechte Mundwinkel bleibt etwas zurück, dabei kann sie weder pfeifen, noch die Backen aufblasen; die Seitwärtsbewegung kann bei den ersten Versuchen ganz gut ausgeführt werden, wird aber nach mehrmaligem Wiederholen immer schwächer, bis es ihr schliesslich ganz unmöglich wird. Beim Versuch, zu lachen, bleiben die Mundwinkel fast ganz unbeweglich, nur die Nase wird stärker nach oben verzogen, so dass ihr Gesicht mehr einen wehmütigen Ausdruck bekommt. Zunge normal, kann nach allen Richtungen ziemlich gut ausgestreckt werden; zeigt keine Atrophie noch fibrilläre Zuckungen. Auch die Zunge zeigt nach öfterem Ausstrecken und Einziehen deutliche Ermüdungserscheinungen. - Ebenso ist es mit der Kaumuskulatur. Die anfangs ziemlich starke Kontraktion des M. masseter und temporalis wird schon nach einigen nach einander gemachten Kaubewegungen sehr schwach, es befällt sie ein Müdigkeitsgefühl, der Mund bleibt kurze Zeit offen, und erst nach einer kürzeren Pause kann sie mit dem Essen fortfahren. Deswegen kann sie auch festere Speisen gar nicht essen. Das Verhalten des weichen Gaumens ist ähnlich: Nach einigen ausreichenden Bewegungen hört die Beweglichkeit beim Intoníeren ganz auf, um nach einigen Sekunden Ruhe wieder zurückzukehren. Rachenreflex schwach auslösbar.

Die Nackenmuskulatur von normaler Entwicklung, die Kopfhaltung auch normal. Sowie sie aber längere Zeit aufrecht sitzt, sinkt ihr Kopf nach vorn oder zurück. Dieselbe schnelle Ermüdung auch bei der Seitwärtsbewegung des Kopfes. Die Schilddrüse ist mässig vergrössert.

Die Muskulatur der Schulter, Brust, oberen und unteren Extremitäten ist gut entwickelt, zeigt nirgends eine Spur von Atrophie, ist aber von auffallend schlaffer Konsistenz, auch bei Kontraktion der Muskeln. Passive Bewegungen überall ausführbar.

Sehnenreflexe ansser dem Tricepsreflex gut auslösbar, so auch die Bauchdeckenreflexe und der Fusssohlenreflex. Kein Clonus. Keine Ataxie. In den Muskeln keine fibrillären Zuckungen. Nervenstämme, Muskeln auf Druck nicht schmerzhaft.

In beiden oberen Extremitäten ist zunächst eine allgemeine 
Schwäche auffallend. Bei aktiver Bewegung kann Patientin ibre Arme nur ungefähr bis zur Horizontalen hében. Bei öfterem Wiederholen dieser Bewegung werden die Exkursionen immer geringer, bis die Arme wie gelähmt herunterhängen and die Kranke die Ärme auch beim besten Willen nicht mehr bewegen kann. Leichte Gegenstände kann sie längere Zeit in der Hand halten, schwere aber muss sie bald wieder niederlegen, da sie ihr sonst nach kurzer Zeit aus der Hand fallen. Grosse Schwierigkeiten bereitet ihr auch das Essen. Den Löffel kann sie nur ein paar Mal nach einander zum Munde führen, während das Schneiden von Fleisch oder Brot nur sehr selten gelingt. Nach einigen schwächlichen Händedruckversuchen werden die nächsten immer schwächer, so dass nach 8-10 maligem Wiederholen die Hand wie gelähmt niedersinkt.

Die sonst gut entwickelte Bauchmuskulatur zeigt dieselbe rasche Ermüdbarkeit. Aus der Rückenlage kann sich Patientin ohne Hilfe ihrer Arme nicht erheben; wiederholt sie aber diese Bewegung, so sinkt sie bald ganz erschöpft zurück, ohne nachher auch die geringste Bewegung machen zu können.

Dasselbe Verhalten zeigen auch die Muskeln der unteren Extremitäten. Nach einigen scheinbar mühelosen Schritten wird der Gang langsamer, das Heben der Beine im Kniegelenk geringer, der Fuss wird auf dem Fussboden geschleift, und Patientin wird von einem grossen Müdigkeitsgefühl befallen und sinkt nach ca. 5-6 Minuten total erschöpft auf den Stuhl.

Was die elektrisehe Untersuchung betrifft, war eine Entartungsreaktion an keinem Muskel nachzuweisen, dagegen war die myasthenische Reaktion Jollys besonders im Facialisgebiet, in der Kaumuskulatur, an Biceps, Unterarmmuskeln, Quadriceps, Pectoralis deutlich vorhanden. An den kurzen Muskeln der Hand konnte sie nur manchmal nachgewiesen werden. Überhaupt war diese Reaktion nicht konstant, sie wechselte nach Tag und Muskeln ziemlich unregelmässig, anscheinend ohne in eigentlichem Zusammenhang mit der grösseren oder geringeren Ermüdbarkeit der Muskeln.

Keine Sensibilitätsstörungen.

Die Sprache ist immer etwas näselnd. Nach einigen deutlich und laut ausgesprochenen Worten wird die Stimme immer leiser und undeutlicher, bis sie schliesslich in ein undeutliches Flüstern übergeht.

Für alle diese Bewegungen war die schnelle Ermüdbarkeit der Muskulatur mit subjektivem Mattigkeitsgefühl charakteristisch. Ruhte aber Patientin eine kurze Zeit aus, so stellte sich wieder die frühere Kraft ein, um bei Wiederholung der Bewegung ebenso rasch, vielleicht etwas schneller wieder zu verschwinden.

Patientin verblieb bis zu ihrem im Jahre 1907 erfolgten Tod auf der Klinik. Über den weiteren Verlauf möchte ich - da er keine wesentliche Abweichung von dem bekannten Verlauf der Myasthenie bot - zusammenfassend berichten.

Besonders drei Momente sind es, die im weiteren Verlauf hervortraten. Erstens die auffallend schnelle zeitliche Schwankung der Krankheit, die sich nicht nur auf längere Perioden - Wochen, Monate - ausstreckte, sondern auch während des Verlaufes eines Tages sehr charakteristisch war. Nach einer gut verbrachten Nacht fühlte sich Patientin morgens viel kräftiger 
als tags vorher, konnte die Augenlider besser heben, die Sprache war deutlich und auch das Gehen fiel ihr viel leichter. Schon nach wenigen Stunden aber senkten sich ihre Augenlider, eine allgemeine Mattigkeit bemächtigte sich ihrer, so dass sie meistens den ganzen Tag im Bett verblieb. Am ausgeprägtesten waren die Symptome abends.

Neben dieser täglichen Schwankung des Krankheitsbildes war das Auftreten von Perioden, wo Patientin ohne nachweisbare Ursache mehrere Wochen, ja monatelang sich viel stärker fühlte und während welcher Zeit sich fast alle Symptome wesentlich besserten, bemerkenswert.

Ohne jede äussere Ursache traten dann, meistens ziemlich plötzlich, Remissionen ein, wobei die oben geschilderten Symptome besonders ausgeprägt wurden. Solche Remissionen kamen im Verlauf ihrer Krankheit öfters wieder, ohne aber einen progredienten Charakter zu zeigen. Auffallend war eine regelmässige Verschlechterung ihres Zustandes während der Zeit der Menstruation.

Eine weitere bemerkenswerte Erscheinung bildeten die bei Myasthenikern so häufig beschriebenen eigenartigen Erstickungsanfälle. Schon in der ersten Zeit ihres klinischen Aufenthaltes bekam Patientin plötzlich Anfälle von starker Atemnot. Sowohl ausserhalb des Bettes als auch beim ruhigen Liegen - im ersteren Falle fiel sie plötzlich zusammen - wurde sie cyanotisch, bekam starke Atemnot, das ganze Gesicht und der Oberkörper wurden mit Schweiss bedeckt, dabei waren die Extremitäten ganz kühl anzufühlen. Der Mund füllte sich rasch mit Speichel und zähflüssigem Schleim und auch mit der grössten Anstrengung konnte sie ihn nicht ausspucken. Dabei war die Zunge nach hinten gesunken und die Atmung ganz oberflächlich, frequent. Der Puls war beschleunigt and filiform, stieg bis über 120. Nach 10-15 Minuten besserte sich der Zustand wieder, besonders wenn man den Schleim aus dem Munde entfernte und die Zunge nach vorn zog. Nach dem Anfall fühlte sie sich immer noch 1-2 Tage sehr matt.

Diese Anfälle wiederholten sich anfangs in Zwischenräumen von mehreren Monaten, später aber wurden sie immer häufiger und nahmen auch an Intensität zu, indem Patientin auch das Bewusstsein verlor und es manchmal eine halbe Stunde dauerte, bis sie unter oftmaliger Anwendung von künstlicher Atmung und Oxygeninhalation zu sich kam. Auch der Exitus trat während eines solchen Anfalles ein, in einem Stadium, wo Patientin sich leidlich fühlte und in jeder Hinsicht eher eine Besserung des Znstandes zu verzeichnen war; auch waren seit dem letzten Anfall mehr als 2 Monate verstrichen. Unter dem oben beschriebenen Bilde bei Bewusstlosigkeit, starker, zunehmender Cyanose, Schweissausbruch und Sekretansammlung im Munde erlag sie dem Anfall trotz künstlicher Atmung und Oxygen in 1 Stunde.

Die Sektion, die 18 Stunden nach dem Exitus gemacht wurde, ergab weder am Gehirn noch an den übrigen Organen etwas Besonderes, weshalb ich es unterlasse, das Sektionsprotokoll ausführlich mitzuteilen. Hervorheben möchte ich nur Folgendes: In der Gegend des linken Frontal- und Parietallappens war eine submeningeale Blutung nachzuweisen, dir ihrem Aussehen nach ganz frisch und so wahrscheinlich eine Folge des so lang andauernden Erstickungstodes war. Am Gehirn und Rückenmark war nichts Pathologisches nachweisbar. Thymus war nicht persistent. Weder in den Lungen 
noch in den Drüsen oder in anderen Organen war eine Tuberkulose nachweisbar. Die Muskulatur schien gut entwickelt, nicht atrophisch, etwas blasser, besonders an den Extremitäten; sonst zeigte sie makroskopisch keine Veränderung.

In den Gefässen überall flüssiges Blut.

Histologisch wurden das zentrale Nervensystem, periphere Nerven, und einige Muskeln untersucht. Vom ersteren untersuchte ich Teile aus den Zentralwindungen, Capsula interna, Brücke, Medulla oblon-

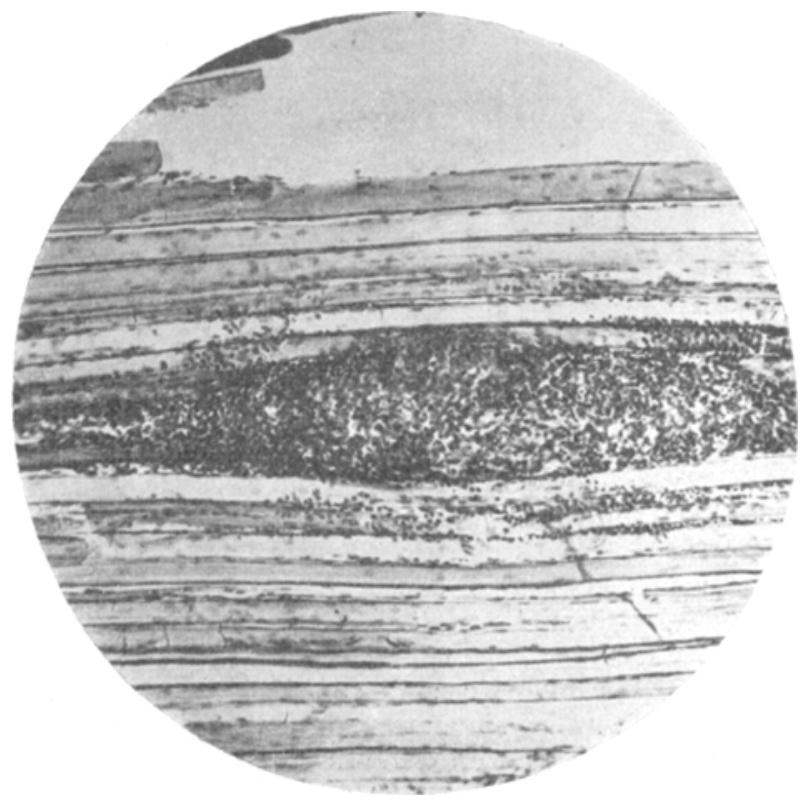

Fig. 1.

Längsschnitt durch den M. vastus med. Spindelförmig angeordnete Zellanhäufung zwischen den Muskelfasern; dieselbe auseinanderdrängend. (Schwache Vergrösserung:)

gata - letztere in iher ganzen Länge - Rückenmark. Die angewandten Methoden waren Weigert-Pal, Marchi, Nissl und van Gieson. Der Befund war in allen Teilen ein negativer. Positiv war nur eine an manchen Stellen ziemlich deutliche Chromatolyse in den Ganglienzellen der Medulla oblongata, die sich aber weder auf gewisse Kerngebiete lokalisierte, noch durch besondere Ausdehnung auszeichnete, dann mikroskopische Blutungen, die sich ebenfalls in der Medulla ablongata vorfanden. Auf diesen letzteren Befund von kleinen Kapillarblutungen möchte ich wegen des Erstickungstodes keinen grösseren 
Wert legen, wobei solche mikroskopisch kleine Blutungen bekanntlich ein häufiger Befund sind; ansserdem machten sie den Eindruck ganz frischer Blutungen, da die roten Blutkörperchen ganz gut erhalten schienen und die Zeichen einer älterén Blutung, wie Hämatoidinkrystalle, Schollen oder mit Pigment beladene Wanderzellen, nirgends nachgewiesen werden konnten. Stärkere Anhäufung von Lymphzellen oder eine Wacherung des Gliagewebes konnte ich nirgends finden.

Ebenso negativ war auch der Befund an den zur Untersuchung

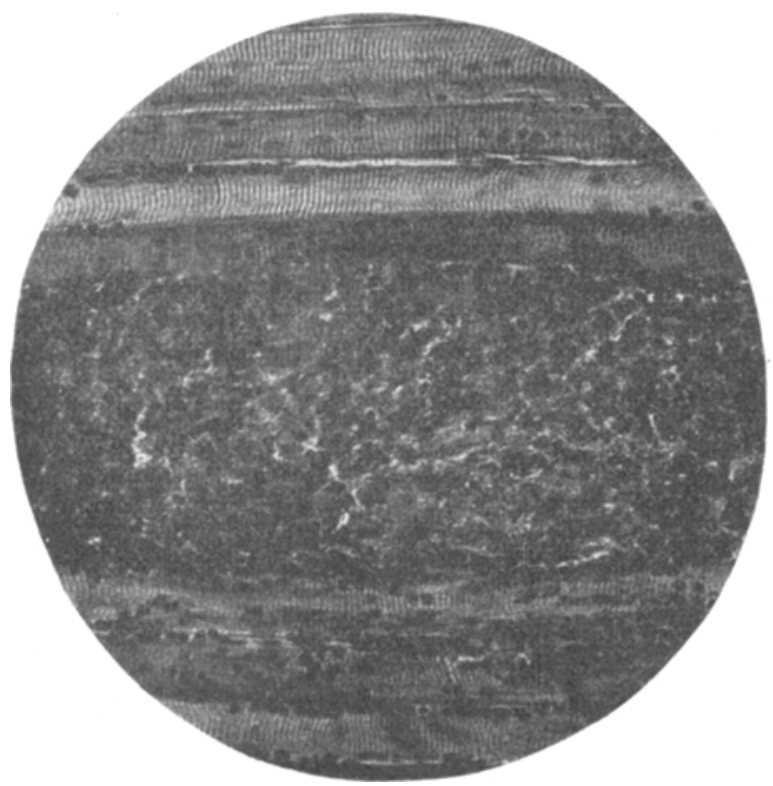

Fig. 2.

Dasselbe. Starke Vergrösserung. Deutliches Erhaltensein der Querstreifung der Muskulatur.

gelangten Nerven: Nn. brachialis, tibialis ant., ischiadicus. Auch die Marchimethode zeigte keinen fettigen Zerfall der Markscheiden.

$\mathrm{Zu}$ positivem Resultate führte jedoch die Untersuchung einzelner Muskeln. Es wurden folgende Muskeln einer näheren histologischen Analyse unterzogen: $\mathrm{Mm}$. biceps von beiden Seiten, masseter, serratus ant., pectoralis, latissimus dorsi, vastus med., sartorius und gastrocnemius. Die Uutersuchung der Herzmuskulatur, des Diaphragma und der Augenmuskeln unterblieb leider. Der Befund war in sämtlichen Muskeln der gleiche, wechselte nur nach Intensität und Anzahl der Veränderungen; eine Ausnabme bildete der M. latissimus dorsi, bei dem 
ausser einer stärkeren Kernwucherung nichts nachgewiesen werden konnte.

Die Muskelbündel zeigen weder in ihrer Dicke noch in ihrer Anordnung eine auffallende Veränderung; während die einzelnen Muskelfasern sich schon wesentlich verschieden verhalten. Obwohl die Querstreifung in den einzelnen Fasern überall gut erhalten ist, ja an manchen Fasern auffallend scharf und deutlich zu sehen ist, färben sich einzelne Muskelfasern, ja sogar nur einzelne Teile derselben ver-

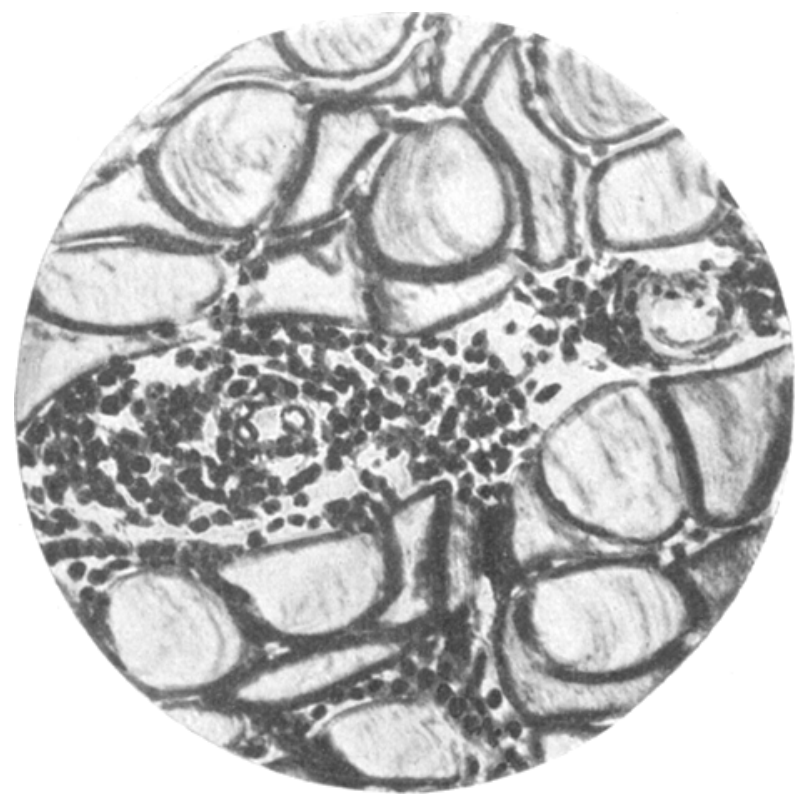

Fig. 3.

Querschnitt durch den M. pectoralis. Zellanhäufung mit quergeschnittenen kapillaren Gefässen. Die perivaskulären Lymphräume sind ebenfalls dicht gefüllt mit Zellen. Zwisehen den Fasern zerstreute Zellen. (Starke Vergrösserung.)

schieden intensiv, das besonders an Längsschnitten, aber oft auch an Querschnitten sehr deutlich nachweisbar ist. Die normal gefärbte Faser geht teils langsam, teils aber plötzlich in die dunkler gefärbte Partie über, die zuweilen - besonders an Längsschnitten - auch etwas verdickt erscheinen, ohne aber an der Querstreifung eine sichtbare Änderung zu erfahren. Unter den verhältnismässig gleich dicken Muskelfasern finden wir in auffallend grosser Zahl ganz dünne Fasern, die bloss $1 / 2$ bis $1 / s$ der normalen Dicke messen. Die Querstreifung ist aber auch an noch so dünnen Fasern immer noch deutlich zu sehen. 
Die Kerne der Muskelfasern haben überall eine normale Lagerung, d. h. sie liegen immer an der. Peripherie der Fasern dicht unter dem Sarkolemm; auffallend ist aber an manchen Stellen eine starke Vermehrung der Kerne; diese Vermehrung muss schon deshalb als pathologisch betrachtet werden, da bekanntlich eine Kernvermehrung normal nur bei älteren Lenten vorkommt, in diesem Falle aber es sich um ein junges Individuum von 23 Jahren handelt.

Die einzelnen Muskelbündel werden durch kleinere und grössere

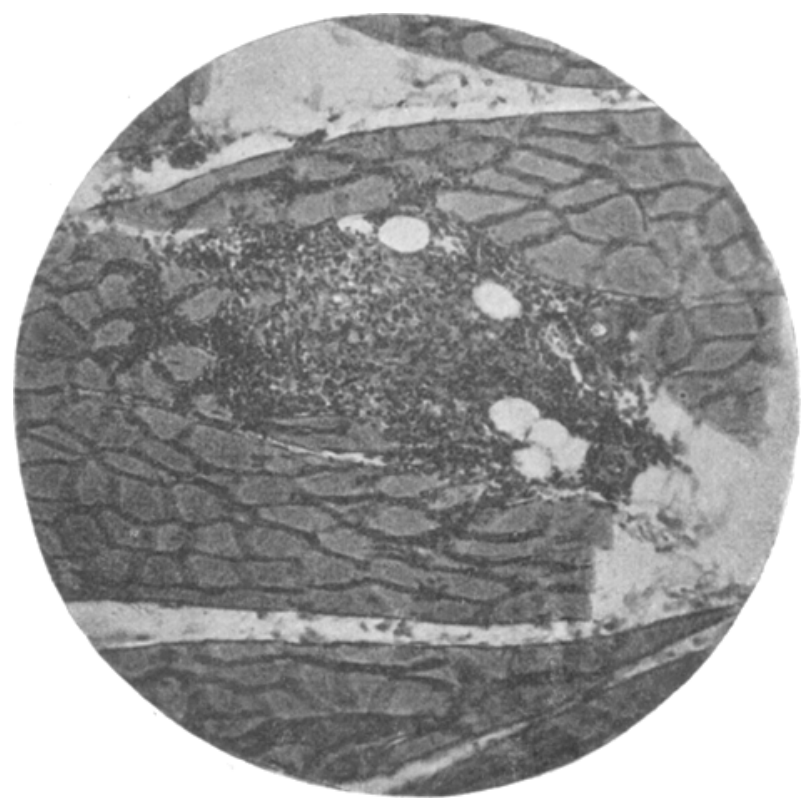

Fig. 4.

Querschnitt durch den M. gastrocnemius. Zellherd mit zwischen die Fasern hineinwuchernden Zellen. Einige Fettzellen an Stelle zugrunde gegangener Muskelfasern. (Schwache Vergrösserung.)

Fettablagerung von einander getrennt; ausserdem findet man aber in einzelnen Bündeln an ganz ungewohnter Stelle zwischen den Fasern einzelne Fettzellen, die ihrer Lage nach wahrscheinlich zugrunde gegangene Muskelfasern ersetzen.

Viel auffallender sind aber die Veränderungen des Bindegewebes. Sowohl an Quer- als auch an Längssehnitten findet man im Verlaufe des Perimysium int. kleine runde Zellen von der Grösse der Lymphzellen, die einen sehr schmalen Protoplasmasaum und einen chromatinreichen Kern besitzen, welcher fast die ganze Zelle ausfüllt. Sie liegen 
teilweise zerstreut in Reihen dem Perimysium int. entlang, teilweise aber bilden sie kleinere und grössere Zellanhäufungen, wobei die einzelnen Muskelfasern auseinandergedrängt werden, manchmal bei grösseren Anhäufungen auch eine Strecke weit degenerieren, zerreissen und Schollen bilden. Eine solche Zellanhäufung nimmt dann häufig an Längsschnitten eine spindelförmige Gestalt an. Von diesen Anhäufungen aus sieht man nun ejnzelne Zellen sich zwischen die Muskelfasern dem Bindegewebe entlang hineinwuchern. In der Mitte der meisten dieser

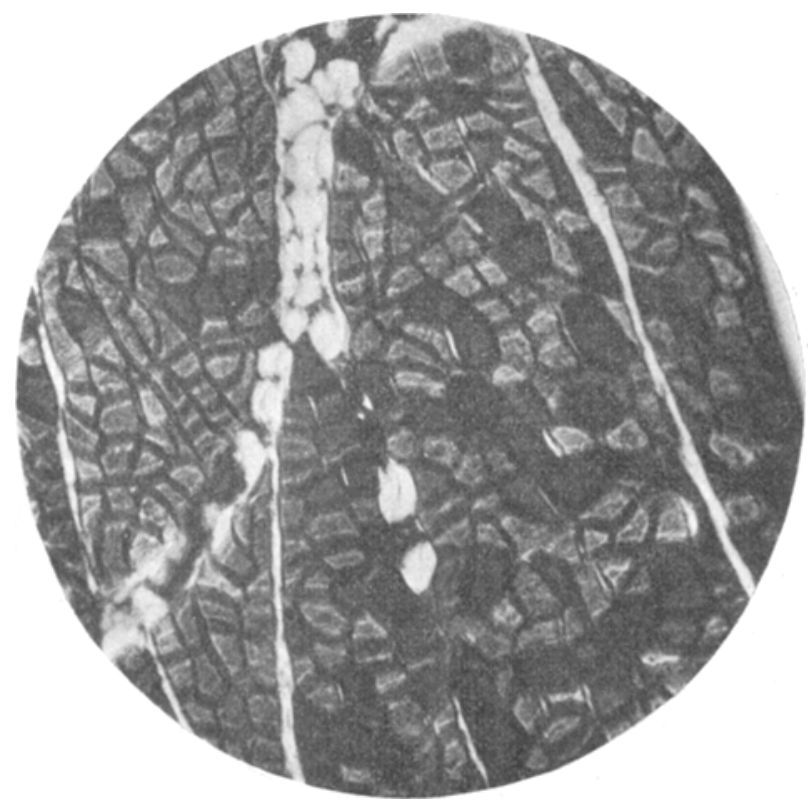

Fig. 5.

Querschnitt durch den M. pectoralis. Verschiedene Färbbarkeit der einzelnen Muskelfasern und Fettzellen.

Zellnester findet man am Querschnitt ein, manchmal auch mehrere kleine kapillare Gefässe, deren Lumen mit Blut stark gefüllt ist und deren perivaskulären Lymphräume mit den gleichen Zellen ebenfalls vollgestopft sind. Auffallend ist manchmal eine Verdickung der Adventitia der Gefässe. Auch an Längsschnitten können diese kleinen Gefässe in den Zellanhäufungen nachgewiesen werden, jedoch viel seltener, da sie doch nicht immer in der Schnittebene liegen. Auch um grössere Gefässe herum findet man diese Zellen, die sie dann manchmal nur an einer Seite, manchmal aber auch ganz umgeben und bei denen die adventitiellen Lymphräume ebenfalls erweitert und vollgepfropft sind. 
Neben diesen einkernigen Zellen konnte ich polynukleäre Leukocyten in keinem der durchsuchten Präparate finden. Einzelne Zellen erreichen manchmal doppelte oder dreifache Grösse der Lymphocyten. Sie haben einen breiteren Protoplasmasaum, haben eine unregelmässige Form oder sind spindelförmig, so dass sie an epitheloide Zellen und an Fibroblasten erinnern. Trotz sorgfältiger Untersuchung konnte ich nirgends Riesenzellen finden.

Wie schon oben erwähnt, findet man um die grösseren Herde unregelmässige, homogene, keine Kernfärbung zeigende Schollen, die wahrscheinlich von zerfallenen Muskelfasern herstammen. An den meisten Stellen verdecken aber diese Zellen diese Schollen ganz, so dass man nirgends grössere Herde von Coagulationsnekrose sehen kann.

Von den Herden aus wuchern diese Zellen sehr weit zwischen die Muskelfasern hinein und bilden um die einzelnen Fasern herum ein unregelmässiges, zusammen hängendes Netzwerk von verschiedener Dicke. Neben dieser zelligen Infiltration, als deren Resultat sieht man an manchen Stellen die zu Fibroblasten umgewandelten Zellen und ein ziemlich dickes Bindegewebe, das an einzelnen Stellen in grosser Ausdehnung manche Fasern in der Form eines sich intensiv färbenden Bindegewebsringes umgibt. Manche dieser Stellen zeigen ausser diesem stark vermehrten und gewucherten Bindegewebe gar keine zellige Infiltration mehr. Auch scheint es mir, dass an diesen Stellen die einzelnen Fasern schmäler sind wie an anderen von Bindegewebe freien Stellen; doch kann ich das nicht bestimmt behaupten, da die Dicke der einzelnen Fasern schon in gesunden Muskeln sehr verschieden ist.

Es sei noch erwähnt, dass in einigen Muskeln, so im M. biceps, serratus die als Muskelspindeln beschriebenen Gebilde auffallend häufig zu sehen sind. Sie bestehen aus 6-8 dünneren, sich intensiver färbenden, mit deutlichen Kernen versehenen Muskelfasern, die von einem festen bindegewebigen Ring umgeben sind. In den meisten sieht man noch neben Gefässen auch einzelne Nervenfasern.

Diesem negativen Befund am Nervensystem gegenüber steht nun seit der Veröffentlichung Weigerts der positive Befund an den Muskeln. Erst seit dieser Weigertschen Veröffentlichung begannen die Autoren auch dem Muskelapparat mehr Aufmerksamkeit zu schenken, und so ist es erklärlich, dass solche Fälle erst in der neuesten Zeit und zwar immer häufiger veröffentlicht werden. Ausserdem kommt es manchmal vor, dass man viele Schnitte, ja sogar manchmal ganze Muskelstückchen umsonst durchsehen muss, bis man darin sicher pathologisch zu deutende Veränderungen findet.

In auffallend vielen Fällen fand man daneben eine teils persistente, teils den Ausgang einer geschwulst- - meist Sarkom-bildenden Thymus. 
Schon beim Weigertschen Fall war eine Thymusgeschwulst vorhanden. Einen ähnlichen, mit Lymphosarkom verbundenen Fall publizierte dann im Jahre $1904 \mathrm{Hun}^{18}$ ), der aber in seiner Arbeit darauf hinwies, dass es sich wahrscheinlich um ein Lymphosarkom des Mediastinum handelte. Fälle, bei denen zwar persistente Thymus nachweisbar war, aber ohne Anzeichen einer Geschwulstbildung, veröffentlichten Link, Burr - letzterer mit Abszessbildung in der Thymus - Steinert und Buzzard in 2 Fällen.

Goldflam ${ }^{17}$ ) fand in einem dem Lebenden exzidierten Muskelstückchen zellige Infiltration der Muskulatur. Bei der nach 2 Jahren erfolgten Autopsie fand sich ein Lymphosarkom der Lunge vor, so dass er den Muskelbefund als eine Metastase des Tumors auffasste, obwohl eine mikroskopische Untersuchung des letzteren unterblieb.

Hier möchte ich auch einen von Oppenheim ${ }^{15}$ ) im Jabre 1899 veröffentlichten Fall erwähnen, den er selbst zu den zweifelhaften Fällen rechnet, bei dem die Sektion ein Lymphosarkom der Thymus aufwies. Die Muskulatur wurde nicht untersucht. Einen bemerkenswerten Befund bildet aber ein „übermässiger Zellreichtum in der den Aquaeductus Sylvii umgebenden grauen Substanz, die von grösstenteils einkernigen Zellen gebildet, in dichten Haufen liegenden Massen auftreten, und zwar sowohl in mehr diffusen als auch in einer herdförförmigen Anordnung, kleine Haufen und Gruppen bildend. Im hinteren Vierhügel sind solche Zellgruppen auch in der Raphe unterhalb des hinteren Längsbündels in der grauen Substanz vorhanden". Auf diesen Befund möchte ich später noch einmal zurückkommen. Fälle, bei denen nur eine persistente Tlyymus nachweisbar war, publizierten z. B. HödeImoser ${ }^{29}$ ), Gruner ${ }^{30}$ ), D uprez-Pagniez ${ }^{31}$ ) u. a., so dass der Gedanke sehr nahestehend schien, zwischen Myas'henie und Thymuspersistenz einen ursächlichen Zusammenhang zu vermuten. Ja man ging so weit, die Frage aufzuwerfen, ob das ganze Krankheitsbild nicht die Folge einer Intoxikation wäre, die durch Stoffe verursacht wärde, die von der persistenten, bezw. pathologisch veränderten Thymus ausgeschieden werden. Obwohl diese Annahme auf keine experimentelle $W$ eise erwiesen werden konnte, so hatte sie, wenn man nur die obigen Befunde in Betracht zog, viel Plausibles. Wenn sie aber richtig wäre, so müsste man wohl in jedem Falle eine persistente oder krankhaft veränderte Thymus antreffen. Dies wurde aber in sehr viel Fällen nicht nachgewiesen. Denn ausser den oben angeführten Fällen gibt es noch einige in der neueren Literatur, bei denen wohl dieselbe zellige Infiltration der Muskeln zugegen war, wo aber weder eine persistente Thymus, noch irgend welche andere Organveränderungen - soweit es nämlich auch mikroskopisch untersucht wurde - nachweisbar 
waren. Dies sind die Fälle von Boldt, Osann, Buzzard (3 Fälle), Knoblauch, Marburg, Frugoni, Mandelbaum und Celler, zu denen ich auch den von mir mitgeteilten rechnen möchte.

Alle diese Fälle, die nach ihrem klinischen Verlauf sicher zur Myasthenie zu rechnen sind, scheinen aber deutlich darauf hinzuweisen, dass eine Persistenz der Thymus oder eine pathologische Veränderung derselben wohl nicht als ein beständiger Befund bei dieser Krankheit anzutreffen ist, und dass alle Versuche, die Krankheit als eine durch die persistente oder pathologisch veränderte Thymus verursachte Intoxikation aufzufassen, wohl nicht genügend begründet werden können.

Hier möchte ich eine andere in neuester Zeit von Chvostek ${ }^{35}$ ) aufgestellte Hypothese erwähnen, die zwar auf rein theoretischer Grundlage aufgebaut ist, welche aber besonders darum Beachtung verdient, da er die Krankheit zum ersten Mal mit anderen Krankheiten in eine Familie vereinigt und den weiteren Forschungen dadurch eine neue Richtung gibt. Er reiht die Myasthenie in die Gruppe der Blutdrüsenerkrankungen ein und will sie durch eine pathologische Funktion der Epithelkörper hervorgerufen wissen. In jeder Beziehung bildet die Tetanie das diametral entgegengesetzte Krankheitsbild der Myasthenie, und so meint er denn, dass, während die Tetanie durch eine Hypofunktion der Epithelkörper verursacht wird, bei der Myasthenie eine Hyper- oder Dysfunktion dieser Drüse vorhanden sein muss.

Auch Lundborg steht schon vor Chvostek auf dem Standpunkt, dass Tetanus, Myoklonie, Myotonie, Paralysis agitans und Myasthenie eine Gruppe ron Erkrankungen bilden, denen je eine Funktionsstörung der Epithelkörper zugrunde liegen könnte. Jedenfalls ist es eine auffallende und schon lange bekannte Tatsache, dass sich zur Myasthenie öfter Basedowsche Krankheit hinzugesellt oder wenigstens einzelne Symptome derselben deutlich nachweisbar sind. Auch bei unserem Falle waren einige Symptome vorhanden, so eine mässige Struma, Exophthalmus, Schlaflosigkeit und manchmal Herzklopfen mit erhöhter Herzaktion. Ich möchte hier nur die Fälle noch von Oppenheim, Jendrassik ${ }^{36}$ ), Löser ${ }^{37}$ ) u. a. erwähnen. Jedenfalls wäre es nicht undenkbar, dass bei einer Erkrankung der Epithelkörper auch die Thyreoidea miterkrankt, besonders wenn man bedenkt, in wie topographisch nahen Beziehungen die zwei Drüsen gerade beim Menschen stehen.

So interessant und verlockend auch die Hypothese Lundborgs und Chrosteks ist, so entbehrt sie bis jetzt jeder anatomischen und experimentellen Grundlage, so dass erst weitere Untersuchungen zeigen müssen, in wie weit sie stichhaltig ist. -

Aus der bis jetzt erschienenen und mir zugänglichen Literatur 
konnte ich somit, inklusive den hier veröffentlichten Fall, 18 Fälle zusammenstellen, bei denen im Muskelapparat eine mehr oder weniger ausgeprägte. aber wohl sicher vorhandene kleinzellige Infiltration der Muskulatur nachweisbar war. Höchstens in den Fällen von Weigert, Goldflam und Hun kann der Muskelbefund als fraglich angesehen werden, da in allen 3 Fällen ein Tumior und zwar ein Sarkom der Thymus vorhanden war und so die Zellanhäufungen - wie es auch von den Autoren angenommen wurde - als Metastasen des Tumors angesehen werden können. Sehr wahrscheinlich scheint mir aber die Annahme, dass man es hier mit rom Tumor ganz unabhängigen Veränderungen zu tun, hatte und dass sie mit den in den anderen Fällen gefundenen Befunden als identisch angesehen werden können.

Es scheint mir nämlich unwahrscheinlich, dass ein Lymphosarkom, welches zur Metastasenbildung führt, sich gerade nur auf das Muskelsystem beschränken würde, wäbrend alle anderen Organe frei blieben. Ausserdem konnten im Falle Goldflams dieselben Zellen 2 Jahre vor dem Exitus nachgewiesen werden. Im Falle Weigerts dauerte die ganze Krankheit jahrelang, im Falle Goldflams sogar 9 Jahre! Wenn diese Zellen nun wirklich Metastasen des Tumors gewesen wären und wir diesen Befund mit der Myasthenie in ursächlichen Zusammenhang bringen, so müssten wir annehmen, dass schon im Anfang der Erkrankung - also vor 2, resp. 9 Jahren - die ersten Metastasen im Muskel auftraten, und dass überhaupt schon damals ein Sarkom der Thymus oder Lunge vorhanden war. Bei dem bekannt rasch zum Exitus führenden Verlauf der Lymphosarkome ist diese Annahme höchst unwahrscheinlich.

Bei näherem Vergleich der mikroskopischen Befunde in den einzelnen Fällen findet man nun, dass sie fast in jeder Hinsicht einander gleich sind: Anhäufung von kleinen einkernigen Zellen in kleineren und grösseren Gruppen, ron wo sie dann einzeln und in Reihen zwischen die Muskelfasern hineindringen. Ihre fast regelmässig um kleine Gofässe gruppierende Anordnung mit Füllung der perivaskulären Lymphräume veranlasste auch viele Autoren zur Benennung "perivaskuläre Infiltration". Nur sehr selten findet man einige endotheloide Zellen, die meiner Meinung nach wohl den manchmal in die Schnittebene fallenden Gefässendothelien entsprechen.

Wohl die meisten Autoren weisen besonders darauf hin, dass ausser dieser Zellinfiltration die Muskulatur im übrigen normal erscheint, ja sogar die Querstreifung noch viel deutlicher zu sehen ist als wie sonst. Schon aus dem oben mitgeteilten mikroskopischen Befund ist es ersichtlich, dass dem nicht ganz so ist. Auffallend war an manchen Stellen ein gewiss nicht physiologischer Dickenunterschied einzelner 
Fasern, Kernvermehrung des Sarkoplasma, bindegewebige Wucherungen und eine Zunahme des Fettgewebes und Auftreten desselben an Stelle zugrunde gegangener Muskelfasern. Wohl waren besonders diese letzteren Veränderungen sehr spärlich, konnten aber mit Sicherheit nachgewiesen werden.

Bei der Durchsicht der in der Literatur beschriebenen Fälle fanden sich in einigen Fällen Bemerkungen, die auf Veränderungen der Muskelfäsern selbst hindeuten. So fanden Goldflam und Link neben den Lymphorrhagien "geschrumpfte" atrophische Fasern. Liefmann ${ }^{32}$ ) beschreibt bei seinem Fall eine Atrophie der rechten Zungenhälfte. Mikroskopisch zeigte sich eine bedeutende Vermehrung des interstitiellen Fett- und Bindegewebes und starke Kernvermehrung in den Muskelfasern. Dejerine u. Thomas ${ }^{33}$ ) fanden eine körnige Degeneration in einzelnen Muskelfasern der Zunge und des Larynx. Frugoni und Marburg fanden fettige Degeneration, ersterer auch Verschmälerung einzelner Fasern. Steinert beschrieb leichte Granulierung des Parenchyms, die nach Formolhärtung wieder verschwand. Tilney ${ }^{34}$ ) fand Dickenzunahme einzelner Muskelfasern mit Vermehrung der Sarkolemmkerne. Buzzard fand zwar in 4 Fällen die Fasern, ausser einer geringeren Färbbarkeit einzelner, normal; in einem Falle aber, der sich durch einen aussergewöhnlich raschen Verlauf auszeichnete, war eine deutliche Degeneration einzelner Fasern nachweisbar, die an manchen Stellen hyalinen, an anderen körnigen Charakter hatte. Auch sah er Vakuolenbildung und Vermehrung der Sarkolemmkerne. Bei einem Falle von Sossedorf war mikroskopisch eine Vermehrung des Fettgewebes der Zunge nachweisbar mit Zeichen der Entzündung und Atrophie, Verschmälerung einzelner Fasern mit Andeutung von Längsstreifung, Kernvermehrung und einzelne leere Sarkolemmaschläuche.

Ausser diesen mikroskopisch nachgewiesenen, zwar geringfügigen Veränderungen finden wir besonders in der älteren Literatur auffallend häufig Fälle, bei denen eine Atrophie einzelner Muskeln oder Muskelgruppen nachweisbar war. Ich will nicht alle diese Fälle einzeln vorführen, möchte nur darauf hinweisen, dass dabei die verschiedensten Muskeln ohne jede Anordnung beteiligt waren, auffallend häufig aber die Zunge, bei der auch sehr oft fibrilläre Zuckungen beobachtet wurden. Wohl sind unter diesen Fällen einige, die Oppenheim in seiner Monographie unter den zweifelhaften Fällen angeführt hat; doch selbst in den von ihm zu den sicheren Fällen gerechneten waren Muskelatrophien vorhanden. Trotzdem kommt er daselbst zur der Konklusion, dass "die Muskelatrophie nicht zu den Symptomen dieser Krankheit gehört".

Diese in letzter Zeit immer zahlreicheren Untersuchungen zeigen 
nun, dass manchmal zwar ein sehr geringer Degenerationsprozess in einzelnen Muskeln doch nachweisbar ist, dèr in Verschmälerung der Fasern, in hyaliner, körniger Entartung, Fettablagerung usw. besteht. Wenn wir uns nun auf den Standpunkt stellen, dass es sich bei dieser Krankheit um irgend eine Intoxikation, sei es von seiten einer pathologisch veränderten Thymus oder Parathyroidea oder einer anderen Ernährungsstörung im Muskel handelt, so scheint es mir ganz selbstverständlich, dass gegen solche schädliche Einwirkung einzelne Muskeln und Muskelfasern nicht immer standhalten können, und dass die weniger widerstandsfähigen an dieser schädlichen Wirkung langsam zugrunde gehen. Da aber die Wirkung manchmal sehr chronisch ist und, wie es aus dem klinischen Verlauf ersichtlich, sehr an Intensität wechseln kann, so ist es klar, dass es nur selten und auch dann erst im späteren Stadium und in den verschiedensten Muskeln zur sichtbaren Atrophie und zu mikroskopisch nachweisbaren Faserveränderungen kommt. Eine leichte Zunahme des Bindegewebes, auffallend starker Unterschied in der Dicke einzelner Fasern, Ausfall und Degeneration einzelner Fasern, Kernwucherung zeigen darauf hin, dass der Muskel langsam geschädigt wird.

Es ist wohl merkwürdig, dass solche Veränderungen bis jetzt nicht in allen Fällen nachgewiesen werden konnten. Dies könnte man meiner Ansicht nach besonders durch zwei Momente erklären. Erstens sind die oben beschriebenen Muskelbefunde im allgemeinen ziemlich geringfügig und können erst manchmal nur nach längerem Suchen gefunden werden, da man sie ausserdem nicht in jedem Muskelstïckchen finden kann. Zweitens glaube ich, dass die meisten dieser Veränderungen, besonders aber die zellige Infiltration - mit Ausnahme z. B. der Bindegewebswucherung - in direktem Zusammenhang stehen mit dem Verlauf der Krankheit; in Zeiten der Besserung gehen die zelligen Anhäufungen zurïck, die geschädigten Muskelfasern regenerieren wieder, so dass bei der mikroskopischen Untersuchung nichts nachweisbar ist. Bei Verschlimmerung des Zustandes treten sie wieder auf und, wenn der Verlauf chronisch ist und die fortwährende Schädigung der Muskelfasern schliesslich zu deren Zerfall führt, so tritt dann die oben geschilderte bindegewebige Wucherung zwischen den Fasern auf, Ein diese Anschaung stützender Fall ist der von Buzzard, der sich durch seinen akuten Verlauf auszeichnete und bei dem eine auffallend deutliche Veränderung an den Muskelfasern zu sehen war.

Gerade für die Wahrscheinlichkeit einer Ernährungsstörung, resp. einer Erkrankung des lymphatischen Systems sprechen auch die mikroskopischen Befunde von Buzzard, der ausser den Muskeln nicht nur im Zentralnervensystem, sondern auch in anderen Organen, wie in 
den Nieren, Nebennieren, in der Leber, Thyreoidea, dieselbe lymphocytäre Infiltration nachweisen konnte. Ähnlichen Befund ergab auch der oben zitierte Fall von Marburg; - auch der Fall von Oppenheim - Zellige Infiltration in der grauen Substanz um den Aquaeductus Sylvii könnte als bierher gehöriger Befund betrachtet werden.

Ich möchte noch einzelne, vielleicht gerade in dieser Richtung bemerkenswerte Beobachtungen einiger Autoren erwähnen. So fand Sitsen eine Lenkocytose des Blutes, zwar mit Überwiegen der polynukleären Zellen, sowie Zellanhäufungen in der Leber und der vergrösserten Milz. Leider war mir die Arbeit nur im Referat zugänglich, so dass ich über nähere Angaben betreffs der übrigen Befunde nicht berichten kann. Auch in einem Falle von Raymond-Lejonne waren die Lymphocyten im Blute vermehrt. Ob nun dies durch ein chemotaktisch wirkendes Gift verursacht wird, welches durch irgend eine pathologisch veränderte Drüse sezerniert wird (Sitsen, Chvostek), oder dass an eine allgemeine Störung in der Lymphzirkulation zu denken ist, kann nach unserem bisherigen Wissen nicht entschieden werden. Jedenfalls scheint es mir sehr wichtig, bei Autopsien und mikroskopischen Untersuchungen auch auf diese Gesichtspunkte zu achten und ausser den Muskeln auch die anderen Organe, besonders aber das Lymphgefässsystem genauer zu untersuchen.

Die zum Teil positiven Befunde in anderen Organen ausserhalb des Muskelsystems würden darauf hinweisen, dass es sich um eine Veränderung handelt, die den ganzen Organismus mehr oder weniger gleichmässig befällt. Die Muskelveränderung wäre also keine Metastasenbildung einer pathologisch verïnderten Thymus, ebensowenig, wie die Erkrankung dieser Drüse, die in einigen Fällen von Myasthenje gefunden wurde, nicht als Ursache dieser Krankheit aufzufassen wäre.

Alle diese aufgezählten Befunde aber sind Tatsachen, die, wie wir sahen, zur Aufstellung mancher Hypothesen ermuntern. Wenn wir uns aber über die wahre Ursache dieser merkwürdigen Krankheit fragen, so müssen wir sagen, dass wir noch immer in vielen Hinsichten im Unklaren sind. Der einzige Fortschritt der letzten Jahre ist, dass wir eine zwar meiner Meinung nach auch nur sekundäre Veränderung im Muskelsystem kennen gelernt haben, die uns aber von dem bisher verfolgten Wege in so weit ablenkt, dass wir die Ursache der Krankheit nicht, wie bisher, im Zentralnervensystem suchen, sondern entweder im Muskel selbst oder in den die Funktion der Muskeln im allgemeinen zu beeinflussen scheinenden Sekretionsprodukten verschiedener Organe, besonders aber in einer Veränderung des Stoffwechsels. Ob da auch andere Faktoren, wie z. B. eine gewisse Minderwertigkeit des Organismus, eine Rolle spielen, darïber kann nichts Sicheres gesagt werden. Jeden- 
falls sind die bei vielen Fällen nachgewiesenen Entwicklungsanomalien auffallend. Diese Gesichtspunkte sind es, die nach den neueren Untersuchungen bei der Aufklärung der Ätiologie dieser Krankkheit bei den weiteren Untersuchungen zu neuen Resultaten führen werden.

\section{Literatur.}

1) Wilks, Guys Hosp. Reports 1877. Vol. XXII. (Zitiert nach Oppenheim.)

2) Erb, Arch. f. Psych. 1879. Bd. 9.

3) Oppenheim, Virch. Arch. 188\%. Bd. 108.

4) Goldflam, Neur. Zentralbl. 1891 u. Deutsche Zeitschr. f. Nervenheilkunde. Bd. 4.

5) Jolly, Berl. klin. Wochenschr. 1895.

6) Strümpell, Deutsche Zeitschr. f. Nervenheilkde. 1896. Bd, 8.

7) Hoppe, Berl. klin. Wochenschr. 1892.

8) Eisenlohr, Deutsche med. Wochenschr. 1893.

9) Senator, Neurol. Zentralbl. 1892.

10) Shaw, Brain 1890.

11) Dreschfeld, Brit. med. Journal 1893.

12) Sossedorf, Genère 1896 (zitiert nach Oppenheim).

13) Schüle, Münch. med. Wochenschr. 1899.

14) Raymond, Leçons sur les maladies du syst. nerv. 1900. VI.

15) Oppenheim, Die myasthenische Paralyse. Berlin 1901.

16) Laqueur-Weigert, Neur. Ztrbl. 1901.

17) Goldflam, Neur. Ztrbl. 1902.

18) Hun, Albany med. Annal. 1904. (Ref.)

19) Steinert, Deutsche med. Wochenschr., Vereinsbeil. 1906.

20) Link, Deutsche Zeitschr. f. Nervenheilkde. 1903. Bd. 23.

21) Boldt, Monatsschr. f. Psych. u. Neur. 1906. Bd. 19.

22) Burr, The Journ. of nerv. and ment. Diss. 1905. (Ref.)

23) Buzzard, Brain 1905.

24) Knoblauch, Arch. f. Psych. 1907.

25) Frugoni, Riv. critic. di clin. med. 1907. (Ref.)

26) Marburg, Zeitschr. f. Heilkde. 1907.

27) Osanu, Monatsschr. f. Psych. u. Neur. 1906.

28) Mandlebaum n. Celler, The Journ. of exper. Medicine 1908.

29) Hödlmoser, Zeitschr. f. Heilkde. 1902. 33.

30) Gruner, Inaug.-Diss. Königsberg (Ref.).

31) Duprez-Pagniez, Nour. Iconogr. de la Salp. 1905. (Ref.)

32) Liefmann, Deutsche Zeitschr. f. Nervenheilkde. Bd. 21.

33) Dejerine-Thomas, Revue neurologique 1901.

34) Tilney, Neurographs I. 1907. (Ref.)

35) Chvostek, Wiener klin. Wochenschr. 1908.

36) Jendrássik, Arch. f. Psych. 1886. Bd. 17.

37) Loeser, Zeitschr. f. Augenheilkde. 1904. 12. 